Power Values of Divisor Sums

Author(s): Frits Beukers, Florian Luca, Frans Oort

Reviewed work(s):

Source: The American Mathematical Monthly, Vol. 119, No. 5 (May 2012), pp. 373-380

Published by: Mathematical Association of America

Stable URL: http://www.jstor.org/stable/10.4169/amer.math.monthly.119.05.373

Accessed: 15/02/2013 04:05

Your use of the JSTOR archive indicates your acceptance of the Terms \& Conditions of Use, available at http://www.jstor.org/page/info/about/policies/terms.jsp

JSTOR is a not-for-profit service that helps scholars, researchers, and students discover, use, and build upon a wide range of content in a trusted digital archive. We use information technology and tools to increase productivity and facilitate new forms of scholarship. For more information about JSTOR, please contact support@jstor.org. 


\title{
Power Values of Divisor Sums
}

\author{
Frits Beukers, Florian Luca, and Frans Oort
}

\begin{abstract}
We consider positive integers whose sum of divisors is a perfect power. This problem had already caught the interest of mathematicians from the 17th century like Fermat, Wallis, and Frenicle. In this article we study this problem and some variations. We also give an example of a cube, larger than one, whose sum of divisors is again a cube.
\end{abstract}

1. INTRODUCTION. Recently, one of the current authors gave a mathematics course for an audience with a general background and age over 50. Although some non-mathematicians have developed a fear and a dislike for mathematics, the course was well attended, and the participants eagerly followed many aspects of pure mathematics. At one occasion, during the discussion of divisor sums, one participant asked: "the sum of the divisors of my age is a square, can you tell me how old I am?" She did not look like she was of age 1 or 3 , or even 22 for that matter, so the teacher answered; "Well I cannot tell you exactly, but I do know your age must be one of 66, 70, 81 or 94". The teacher then asked; "do you think there are infinitely many integers of which the divisor sum is a square?" This was a nice example of the theme of the course, how do mathematicians arrive at their conjectures? Moreover, how do interesting questions stimulate the search for new mathematics? The teacher's question is also the starting theme of this note.

2. THE PROBLEM. By the divisor sum of an integer $n$, we mean the sum of all divisors, including $n$ itself, and we denote it by $\sigma(n)$. Divisor sums are among the oldest quantities studied in number theory. Already in Greek times people were interested in perfect numbers. That is, a number $n$ whose sum of divisors, excluding $n$ itself, equals $n$ again. In terms of the $\sigma$-function this comes down to $\sigma(n)=2 n$. An important feature of $\sigma(n)$ is its multiplicative property. This means that if $m$ and $n$ are co-prime (greatest common divisor one) then $\sigma(m n)=\sigma(m) \sigma(n)$. This reduces the computation of $\sigma(n)$ to a factorization of $n$ and a computation of values of divisor sums of primes or prime powers. Clearly, for a prime $p$ we have $\sigma(p)=p+1$ and for its $k$ th power $\sigma\left(p^{k}\right)=1+p+\cdots+p^{k}$. For a discussion and proof of the multiplicative property of $\sigma$ one can consult most books in elementary number theory, for example [7, Section 16.7]. The following theorem answers the teacher's question.

Theorem 2.1. There exist infinitely many positive integers $n$ such that $\sigma(n)$ is the square of an integer.

We cannot attach the name of a first discoverer of this theorem, but it is very likely that this theorem has been proven many times in the history of number theory. The proof is not hard and will be given in Section 3, but we can already explain its main idea here. We write down the factorizations of $\sigma(p)$ for all primes $p \leq 23$ :

$$
\begin{aligned}
& \sigma(2)=3, \\
& \sigma(3)=2^{2},
\end{aligned}
$$

http://dx.doi.org/10.4169/amer.math.monthly.119.05.373

MSC: Primary 11N25, Secondary 11N64 


$$
\begin{aligned}
\sigma(5) & =2 \times 3, \\
\sigma(7) & =2^{3}, \\
\sigma(11) & =2^{2} \times 3, \\
\sigma(13) & =2 \times 7, \\
\sigma(17) & =2 \times 3^{2}, \\
\sigma(19) & =2^{2} \times 5, \\
\sigma(23) & =2^{3} \times 3 .
\end{aligned}
$$

There are plenty of ways in which we can make a selection of these equalities such that after multiplication the right hand side is a square. For example, multiply $\sigma(2)=3$ and $\sigma(11)=2^{2} \times 3$ to get $\sigma(2) \sigma(11)=2^{2} \times 3^{2}$. By the multiplicative property of $\sigma$ we get $\sigma(22)=2^{2} \times 3^{2}$. Similarly, the factorizations of $\sigma(7)$ and $\sigma(17)$ give us $\sigma(7 \times 17)=2^{4} \times 3^{2}$, again a square. To find more of such relations we symbolize a factorization $2^{a} 3^{b} 5^{c} 7^{d}$ by its so-called exponent vector $(a, b, c, d)$. We then need to find a subset of these vectors whose sum is $(0,0,0,0)$ modulo 2 . In other words, we look for linear relations between the exponent vectors modulo 2. Our problem is thus reduced to linear algebra over the field of two elements, $\mathbb{F}_{2}$. In the example above, the rank of the 9 exponent vectors over $\mathbb{F}_{2}$ is 4 . Hence the space of relations has dimension $9-4=5$ and thus we can construct $2^{5}-1=31$ non-trivial relations in this way. This gives us 31 examples of solutions $m, n$ to $\sigma(n)=m^{2}$. It turns out that by increasing the number of factorizations, the number of values $n$ for which $\sigma(n)$ will increase very quickly.

To continue the teacher's investigations, it is a pleasant surprise to find that there exist integers whose divisor sum is a cube, or a fourth power, or higher powers. The smallest examples of $\sigma(n)=m^{3}$ with $n>1$ are $\sigma(7)=2^{3}, \sigma(102)=6^{3}, \ldots$ and the smallest fourth power example is $\sigma(217)=4^{4}$ (incidently, $4^{4}=2^{8}$ ). We have the following theorem.

Theorem 2.2. Given a positive integer $k$, there exist infinitely many positive integers $n$ such that $\sigma(n)$ is the $k$ th power of an integer.

Although we found a proof of this theorem in the beginning of 2010, the ingredients have been around for a much longer time. So there is a fair chance that it has been observed and proven before. We will give a proof in Section 4. However, there is a much more surprising result. It can be found in a recent preprint by T. Freiberg [5], which deals with the problem of estimating the number of $k$ th powers in products of shifted primes. For the particular case of divisor sums it has the following consequence.

Theorem 2.3 (Freiberg, 2010). Let $k$ be a positive integer and let $s_{k}(x)$ be the number of positive integers $n<x$ for which $\sigma(n)$ is a kth power. Then there exists a positive real number $x_{k}$ such that

$$
s_{k}(x)>x^{0.7}
$$

for all $x \geq x_{k}$.

Naively one might have guessed that $s_{k}(x)$ would be about $x^{1 / k}$, comparable to the number of $k$ th powers below $x$. So this theorem is indeed remarkable. In a slightly 
exaggerated way one might say that the values of $\sigma$ are really eager to be perfect powers. The proof can be found in [5, Theorem 1.1], where an exponent slightly larger than 0.7 is given. We are thankful to the referee for pointing out Freiberg's preprint to us. The paper [2] contains the ingredients for the proof of this theorem. More precisely, [2] deals with square values of the Euler totient function $\phi$, but similar arguments apply to $\sigma$.

The idea behind the proof of the two theorems above is again to write down factorizations of many $\sigma(p)$, with $p$ prime, and choose factorizations from this list whose product is a $k$ th power. In particular take $k=3$ and the factorizations of $\sigma(2), \ldots, \sigma(23)$ given above. This time the use of linear algebra in finding linear relations modulo 3 between the exponent vectors is not going to help us. We are interested only in relations with coefficients 0,1 . Straightforward solution of linear equations modulo 3 would also give us coefficients 2 , which are not useful to us. The following theorem comes to our rescue.

Theorem 2.4. Let $k$ be a prime power and $r$ a positive integer. Then any subset of the additive group $(\mathbb{Z} / k \mathbb{Z})^{r}$ with more than $r(k-1)$ elements contains a non-empty subset whose sum of elements is zero.

This theorem, in the language of abelian groups, was proved independently by Van Emde Boas, Kruyswijk [4] and Olson [10]. Unfortunately [4] is difficult to come by, but in [10] one finds a simple and delightful proof. Application in the case $k=3$ shows that if the number of factorizations exceeds twice the length of the exponent vectors, then there is a relation. In our example there are 8 factorizations with exponent vectors of length 3. So we should be able to find relations. For example, $\sigma(7)=2^{3}$, $\sigma(2 \times 5 \times 11)=2^{3} \times 3^{3}$ and $\sigma(3 \times 17 \times 23)=2^{6} \times 3^{3}$. A computer search shows that we can construct 15 examples of $\sigma(n)=m^{3}$ in this way. We used a computer search this time, because Theorem 2.4 is only an existence theorem. The actual construction of a subset with sum zero can be a very difficult problem, especially for larger $r$, say $r>100$.

In Neil Sloane's online encyclopedia of integer sequences [11] we find several sequences dedicated to this problem, namely A006532 $\left(\sigma(n)=m^{2}\right), \mathrm{A} 020477(\sigma(n)=$ $\left.m^{3}\right), \mathrm{A} 019422\left(\sigma(n)=m^{4}\right), \mathrm{A} 019423\left(\sigma(n)=m^{5}\right), \mathrm{A} 019424\left(\sigma(n)=m^{6}\right), \mathrm{A} 048257$ $\left(\sigma(n)=m^{7}\right)$ and $\mathrm{A} 048258\left(\sigma(n)=m^{8}\right)$.

To top off our story, we turn to a very general question.

Question 2.5. Given positive integers $l \geq 1$ and $k \geq 2$, do there exist integers $n$ such that $\sigma\left(n^{l}\right)$ is a $k$ th power?

In Dickson's History of the Theory of Numbers [3, Vol I, Chapter 2, p. 54] we find that Fermat observed $\sigma\left(7^{3}\right)=20^{2}$ and asked for more examples of $\sigma\left(n^{3}\right)=m^{2}$ and, as second question, $\sigma\left(n^{2}\right)=m^{3}$. Subsequently, Wallis, Frenicle and many others came up with additional examples. Their strategy is similar to the one we used above. Write down the prime factorizations of $\sigma\left(p^{3}\right)$ for all primes $p$ up to a certain limit. By way of example we record the prime factorizations of $\sigma\left(p^{3}\right)$ for all $p<50$ and prime factors less than 50:

$$
\begin{aligned}
& \sigma\left(2^{3}\right)=3 \times 5 \\
& \sigma\left(3^{3}\right)=2^{3} \times 5 \\
& \sigma\left(5^{3}\right)=2^{2} \times 3 \times 13
\end{aligned}
$$




$$
\begin{aligned}
\sigma\left(7^{3}\right) & =2^{4} \times 5^{2} \\
\sigma\left(13^{3}\right) & =2^{2} \times 5 \times 7 \times 17 \\
\sigma\left(17^{3}\right) & =2^{2} \times 3^{2} \times 5 \times 29 \\
\sigma\left(31^{3}\right) & =2^{6} \times 13 \times 37 \\
\sigma\left(41^{3}\right) & =2^{2} \times 3 \times 7 \times 29^{2} \\
\sigma\left(43^{3}\right) & =2^{3} \times 5^{2} \times 11 \times 37 \\
\sigma\left(47^{3}\right) & =2^{5} \times 3 \times 5 \times 13 \times 17
\end{aligned}
$$

The fact that $\sigma\left(p^{3}\right)=(p+1)\left(p^{2}+1\right)$ certainly helps in getting small primes in the factorizations. We have listed 10 factorizations with 9 distinct primes occurring. Hence we can find a subset of factorizations whose product is a square. For example,

$$
\sigma\left((2 \times 3 \times 5 \times 13 \times 41 \times 47)^{3}\right)=2^{14} \times 3^{4} \times 5^{4} \times 7^{2} \times 13^{2} \times 17^{2} \times 29^{2} .
$$

This, by the way, is the smallest example of $\sigma\left(n^{3}\right)=m^{2}$ beyond $\sigma\left(1^{3}\right)=1^{2}$ and Fermat's $\sigma\left(7^{3}\right)=20^{2}$. It was found by Wallis in 1658 (see [3, Vol I, p. 55]). Increasing the number of primes, and the bound on the allowed prime factors, we find many more such possibilities. For example, listing the factorization of $\sigma\left(p^{3}\right)$ for all primes $p<10000$ and prime factors $<2000$ gives us 251 factorizations with 182 distinct primes. This provides us with at least $2^{251-182}-1=2^{69}-1$ examples of $\sigma\left(n^{3}\right)=m^{2}$.

As for Fermat's second question, $\sigma\left(n^{2}\right)=m^{3}$, the smallest example (after $\sigma\left(1^{2}\right)=$ $\left.1^{3}\right)$ is

$$
\sigma\left((2 \times 3 \times 11 \times 653)^{2}\right)=1729^{3},
$$

found relatively late by A.S. Bang in 1878 (see [3, Vol I, p. 56]). This belated discovery has probably something to do with the large prime 653. Several larger examples had been found earlier by Wallis and Frenicle (see [3, Vol I, pp. 55, 56]). To find such examples, one can write down all factorizations of $\sigma\left(p^{2}\right)$ for the first, say $K$, primes $p$ and whose prime factors are less than a relatively small bound, say $B$. For example, taking $K=1000$ and $B=100$ we find 26 factorizations with 12 distinct primes. Since 26 is larger than twice 12, an application of Theorem 2.4 shows that there exists a subset of these factorizations whose product is a cube.

But there is more. Additional computer calculations of a similar kind seem to suggest the following two conjectures.

Conjecture 2.6. For every positive integer $k$ there exist infinitely many positive integers $n$ such that $\sigma\left(n^{2}\right)$ is $k$ th power of an integer.

Conjecture 2.7. For every positive integer $k$ there exist infinitely many positive integers $n$ such that $\sigma\left(n^{3}\right)$ is kth power of an integer.

For example, we found that among the factorizations of the first 10000 primes $p$ there are 280 factorizations of $\sigma\left(p^{3}\right)$ with prime factors $<1000$. The number of distinct prime factors that occur is 125 . So there are more than twice as many factorizations as there are prime factors. Hence, Theorem 2.4 tells us there exists an example of $\sigma\left(n^{3}\right)=m^{3}$. However, finding it is quite a computational challenge. Herman te Riele was kind enough to make such an attempt on our behalf and reported the following in a 
personal communication. He considered 63 factorizations of $\sigma\left(p^{3}\right)$ with prime factors $<200$. The number of primes that occur in the factorizations is 46 . Although Theorem 2.4 does not apply here, Te Riele found nevertheless that $\sigma\left(n^{3}\right)=m^{3}$ with

$$
\begin{aligned}
n= & 5 \times 11 \times 13 \times 17 \times 41 \times 47 \times 193 \times 239 \times 443 \times 499 \\
& \times 701 \times 1087 \times 3583 \times 5507 \text { and } \\
m= & 2^{17} \times 3^{5} \times 5^{6} \times 7 \times 13^{4} \times 17^{2} \times 29 \times 37 \times 61 \times 97 \times 149 \times 157 .
\end{aligned}
$$

It would be interesting to know if this is the smallest solution of $\sigma\left(n^{3}\right)=m^{3}$ with $n>1$. We were unable to find very much on the problem $\sigma\left(n^{l}\right)=m^{k}$ with $k, l \geq 2$ in modern literature. In Neil Sloane's online encyclopedia of integer sequences [11] we find several sequences dedicated to this problem, A008847 $\left(\sigma\left(n^{2}\right)=m^{2}\right)$, A008850 $\left(\sigma\left(n^{2}\right)=m^{3}\right)$ and $\mathrm{A} 008849\left(\sigma\left(n^{3}\right)=m^{2}\right)$.

For any pair $(l, k)$ with $l>3, k>1$ we expect that the number of solutions to $\sigma\left(n^{l}\right)=m^{k}$ is finite.

3. THE CASE OF SQUARE VALUES. We give an elementary proof of Theorem 2.1 which is based on the ideas explained in the previous section. We have seen that finding examples of $\sigma(n)=m^{2}$ is not hard. The only concern is that we like to be certain to find infinitely many such examples. The proof we present here works from scratch and doesn't use any results on the distribution of prime numbers. It is based on an idea of Ronald van Luijk, published anonymously in [9].

Let us denote the sequence of primes by $p_{1}=2, p_{2}=3, \ldots$ Choose a positive integer $N>2$. For every $i$ let $r_{i}$ be the smallest integer such that $p_{i}^{r_{i}}>N$. Clearly $r_{i}=$ 1 if $p_{i}>N$. Choose $t$ such that $p_{t}$ is larger than every prime factor in $\prod_{p_{i} \leq N} \sigma\left(p_{i}^{r_{i}}\right)$. For any $p_{i}>N$ we have $\sigma\left(p_{i}\right)=2\left(p_{i}+1\right) / 2$. Hence, the prime factors of $\sigma\left(p_{i}\right)$ are strictly less than $p_{i}$. Thus we see that every factorization of $\sigma\left(p_{i}^{r_{i}}\right)$ with $i \leq t$ consists of primes strictly less than $p_{t}$. We write down the factorizations

$$
\sigma\left(p_{i}^{r_{i}}\right)=p_{1}^{a_{i 1}} p_{2}^{a_{i 2}} \cdots p_{t-1}^{a_{i, t-1}}, \quad \text { for } i=1, \ldots, t .
$$

The number of prime factorizations is $t$ and the total number of prime factors in these factorizations is strictly less than or equal to $t-1$. So in the $t \times(t-1)$-matrix $\left(a_{i j}\right)$, the number of rows exceeds the length of the rows. Hence, it follows from linear algebra over the field $\mathbb{F}_{2}$ of two elements that we can find $\epsilon_{i} \in\{0,1\}$ with $1 \leq i \leq t$, not all zero, such that

$$
\epsilon_{1}\left(a_{11}, \ldots, a_{1, t-1}\right)+\cdots+\epsilon_{t}\left(a_{t 1}, \ldots, a_{t, t-1}\right)
$$

has even entries. Hence,

$$
\sigma\left(p_{1}^{r_{1}}\right)^{\epsilon_{t}} \cdots \sigma\left(p_{t}^{r_{t}}\right)^{\epsilon_{t}}=\sigma\left(p_{1}^{\epsilon_{1} r_{1}} \cdots p_{t}^{\epsilon_{t} r_{t}}\right)
$$

is a square. Note that we have constructed an example of $\sigma(n)=m^{2}$ where the prime power factors of $n$ are all larger than $N$. By choosing $N$ as big as we like we can construct infinitely many such examples. This proves Theorem 2.1.

4. HIGHER POWERS. As a curiosity we mention the following.

Remark 4.1. Let $k$ be any integer $\geq 2$. If there are infinitely many Mersenne primes (i.e., primes of the form $2^{p}-1$ ) then there are infinitely many integers $n$ such that $\sigma(n)$ is the $k$ th power of an integer. 
Proof. Denote the set of exponents $\left\{p: 2^{p}-1\right.$ prime $\}$ by $\mathcal{P}$. We have assumed that this set is infinite. If we consider the elements of $\mathcal{P}$ modulo $k$, then there is a residue class $a(\bmod k)$ which contains infinitely many elements of $\mathcal{P}$. Choose $k$ elements $p_{1}, \ldots, p_{k} \in \mathcal{P}$ such that $p_{1} \equiv \cdots \equiv p_{k} \equiv a(\bmod k)$. Then clearly, $p_{1}+\cdots+p_{k} \equiv$ $k a \equiv 0(\bmod k)$. Observe that

$$
\sigma\left(\left(2^{p_{1}}-1\right) \times \cdots \times\left(2^{p_{k}}-1\right)\right)=2^{p_{1}} \times \cdots \times 2^{p_{k}} .
$$

The latter number equals $2^{p_{1}+\cdots+p_{k}}$ whose exponent we have seen to be divisible by $k$. Hence we have a $k$ th power value of $\sigma$. Since there are infinitely many choices of $p_{1}, \ldots, p_{k}$, we get infinitely $k$ th power values of $\sigma$. This proves the remark.

However, it is unknown whether there are infinitely many Mersenne primes. So we had better look for another proof of Theorem 2.2. The following proof uses more advanced methods than in the case of square values. One of the ingredients is the prime number theorem.

Theorem 4.2. If $\pi(x)$ is the prime counting function, then

$$
\pi(x) \sim \frac{x}{\log x}
$$

as $x \rightarrow \infty$.

The $\sim$ sign means that the ratio of the functions tends to 1 as $x \rightarrow \infty$. For a proof, see [12]. In addition we need the following.

Proposition 4.3. If $M>1$ is given, then there exists $C>0$ such that the number of primes $p \leq x$, where $p+1$ is divisible by a prime $\geq p / M$, is bounded from above by $\left.C x /(\log x)^{2}\right)$ as $x \rightarrow \infty$.

Proof. The proposition depends on a sieve result which can be found in the book by Halberstam and Richert [6, Theorem 3.12]. This theorem implies that, given an even integer $a$, the number of primes $q<x$, such that $a q-1$ is also prime, is bounded from above by a constant $C_{a}$ times $x /(\log x)^{2}$.

To apply this to our situation, let $p$ with $M<p \leq x$ be a prime such that $\sigma(p)$ has a prime divisor $\geq p / M$. This means that there exists a prime $q$ and an even integer $a<$ $M+1$ such that $p+1=a q$. Certainly $q<x$. From the sieve result, it follows that the number of such pairs $(a, q)$ is bounded from above by $\left(\sum_{a<M+1} C_{a}\right) x /(\log x)^{2}$. Hence, the number of primes $p \leq x$ such that $p+1$ is divisible by a prime $\geq p / M$ is bounded from above by a constant times $x /(\log x)^{2}$.

Proposition 4.3 tells us that the proportion of the primes $p \leq x$ such that $p+1$ is divisible by a prime $\geq p / M$ is bounded from above by $C x /\left(\pi(x)(\log x)^{2}\right)$. So this proportion is bounded from above by $C / \log x$, which goes to 0 when we let $x \rightarrow \infty$. Hence, the number of primes $p \leq x$ whose prime factorization does not contain a prime factor $\geq p / M$ is still $\sim \pi(x) \sim x / \log x$. This is what we will exploit in the following proof.

Proof of Theorem 2.2. Choose $M>k \log k$ and choose $x$ sufficiently large. Write down the factorizations of $\sigma(p)$ for all primes $p$ with $\sqrt{x} \leq p \leq x$. Then delete all factorizations which contain a prime factor $\geq p / M$. According to Proposition 4.3 this 
is only a small fraction of the factorizations and this fraction goes to zero as $x \rightarrow \infty$. So from Theorem 4.2 and Proposition 4.3 it follows that for sufficiently large $x$ we get about $x / \log x$ factorizations which contain at most $(x / M) / \log (x / M) \sim x / M \log (x)$ distinct prime factors. So the number of factorizations exceeds $M$ times the number of prime factors. We now apply a slight generalization of Theorem 2.4 which works for all $k$, rather than just prime power values. It can be found [1, Theorem 2] and it reads as follows.

Theorem 4.4. If $k \geq 3$ and $r$ are positive integers, then any subset of $(\mathbb{Z} / k \mathbb{Z})^{r}$ of cardinality $>r k \log k$ contains a non-empty subset whose sum of elements is $0 \bmod k$.

We apply this theorem to the set of exponent vectors modulo $k$ of our factorizations. These vectors have length about $x /(M \log x)$ and they are $x / \log x$ in number. Hence, as we remarked before, this cardinality exceeds $M$ times the length of the exponent vectors. Since $M>k \log k$, Theorem 4.4 can be invoked and we find a non-trivial relation, and hence a number whose divisor sum is a $k$ th power. By choosing $x$ as large as we like, we can produce infinitely many examples in this way, and Theorem 2.2 is proven.

\section{REFERENCES}

1. W. R. Alford, A. Granville, C. Pomerance, There are infinitely many Carmichael numbers, Ann. of Math. 139 (1994) 703-722; available at http://dx. doi.org/10.2307/2118576.

2. W. D. Banks, J. B. Friedlander, C. Pomerance, I. E. Shparlinski, Multiplicative structure of values of the Euler function, in High Primes and Misdemeanours: Lectures in Honour of the 60th Birthday of Hugh Cowie Williams, Edited by A. van der Poorten, A. Stein, and H. Williams, Fields Inst. Commun., Vol. 41, Amer. Math. Soc., Providence, RI, 2004. 29-47.

3. L. E. Dickson, History of the Theory of Numbers, Vol. I, Divisibility and Primality, Chelsea Publishing, New York, 1966.

4. P. van Emde Boas, D. Kruyswijk, A combinatorial problem on finite Abelian groups, Math. Centrum Amsterdam Afd. Zuivere Wisk. 1967 (1969) ZW-009.

5. T. Freiberg, Products of shifted primes simultaneously taking perfect power values (2010), available at http://arxiv.org/abs/1008.1978.

6. H. Halberstam, H.-E. Richert, Sieve Methods, London Mathematical Society Monographs, No. 4. Academic Press, London-New York, 1974.

7. G. H. Hardy, E. M. Wright, An Introduction to the Theory of Numbers, fifth edition. The Clarendon Press, Oxford University Press, New York, 1979.

8. T. E. Mason, Problems and Solutions, Amer. Math. Monthly 23 (1916) 394; available at http://dx. doi.org/10.2307/2971845.

9. Problem section, Nieuw Archief voor Wiskunde 11 5th series (2010) 149.

10. J. E. Olson, A combinatorial problem on finite Abelian groups I, II, J. Number Theory 1 (1969) 811 available at http://dx.doi.org/10.1016/0022-314X(69)90021-3; and 195-199 available at http://dx.doi.org/10.1016/0022-314X(69)90037-7.

11. N. J. Sloane, The Online Encyclopedia of integer sequences, available at http://oeis . org.

12. D. B. Zagier, Newman's short proof of the prime number theorem, Amer. Math. Monthly 104 (1997) 705-708; available at http://dx.doi.org/10.2307/2975232.

FRITS BEUKERS received his Ph.D. in 1979 at the University of Leiden, Netherlands as a student of Rob Tijdeman. After a one year membership at the Institute for Advanced Study, Princeton, he became an assistant professor in Leiden. He is currently working as full professor at the University of Utrecht. His main research interests range from number theory to arithmetic of linear differential equations, and hypergeometric functions. Departement of Mathematics, University of Utrecht f.beukers@uu.nl

FLORIAN LUCA received a B.S. in Mathematics from the University of Iasi, Romania in 1991 and a Ph.D. in Mathematics from the University of Alaska, Fairbanks in 1996. After a few visiting positions at Syracuse 
University, Bielefeld University in Germany, and the Czech Academy of Sciences in Prague, he accepted an appointment at the Universidad Nacional Autonóma de México in Morelia, Mexico where he is still today. His research interests revolve around questions from elementary number theory and diophantine equations.

Instituto de Matemáticas, Universidad Nacional Autónoma de México, Morelia

fluca@matmor.unam.mx

FRANS OORT did his Ph.D. research mainly in Pisa (under Aldo Andreotti) and in Paris (under Jean-Pierre Serre), and he obtained his Ph.D. degree in 1961 at Leiden University. He has held positions in Amsterdam and in Utrecht, and he has held many visiting positions (such as Harvard, Aarhus, IAS, Kyoto University, MIT, and Columbia University). Although retired since 2000, he is still actively engaged in research in algebraic geometry, more specifically in moduli spaces of abelian varieties in positive characteristic.

Departement of Mathematics, University of Utrecht

f.oort@uu.nl

\section{Irrational Numbers Redux}

In a 1973 MonTHLy Classroom Note [1] called "Irrational Numbers", the following non-constructive short argument was given to show that an irrational number raised to an irrational power may be rational. Consider the identity $\left(\sqrt{2}^{\sqrt{2}}\right)^{\sqrt{2}}=2$. If $\sqrt{2}^{\sqrt{2}}$ is rational, then we are done. If not, then $\sqrt{2}^{\sqrt{2}}$ is irrational, and so $\left(\sqrt{2}^{\sqrt{2}}\right)^{\sqrt{2}}$ serves as an example. It was also observed there along similar lines that an irrational number raised to an irrational power may be irrational, by considering the identity $\sqrt{2}^{\sqrt{2}+1}=\sqrt{2}^{\sqrt{2}} \sqrt{2}$.

These proofs, while being elementary, are non-constructive in that the logical principle of the excluded middle (tertium non datur) is used, which the intuitionists reject. As remarked also in [1], it is known that $\sqrt{2}^{\sqrt{2}}$ is irrational, since it is the square root of Hilbert's number $2^{\sqrt{2}}$, which was proved to be transcendental by Kuzmin [2] in 1930. However, this result is not elementary, and is not used above.

In this note we give a constructive elementary proof of the fact that an irrational number raised to an irrational power can be rational.

First we note that $\log _{2} 3$ is irrational, for otherwise if $\log _{2} 3=\frac{p}{q}$, where $p$ and $q$ are positive integers, then $2^{p}=3^{q}$, which is impossible, as $2^{p}$ is even while $3^{q}$ is odd. Our concrete example is then given by $\sqrt{2}^{\left(2 \log _{2} 3\right)}=3$, which is rational, and $\sqrt{2}$ and $2 \log _{2} 3$ are irrational. Also, $\sqrt{2}^{\log _{2} 3}=\sqrt{3}$ shows that an irrational number raised to an irrational power may be irrational.

\section{REFERENCES}

1. J.P. Jones and S. Toporowski. Irrational numbers. Amer. Math. Monthly, 80 (1973) 423-424.

2. R. Kuzmin. On a new class of transcendental numbers. Izv. Akad. Nauk SSSR, Ser. Mat., 7 (1930) 585-597.

— Submitted by AMOL SASANE, Stockholm, Swenden 